\title{
Table top nanopatterning with extreme ultraviolet laser illumination
}

\author{
M.G. Capeluto ${ }^{\text {c }}$, P. Wachulak ${ }^{\mathrm{a}, \mathrm{b}}$, M.C. Marconi ${ }^{\mathrm{a}, \mathrm{b}, *}$, D. Patel ${ }^{\mathrm{a}, \mathrm{b}}$, C.S. Menoni ${ }^{\mathrm{a}, \mathrm{b}}$, \\ J.J. Rocca ${ }^{\text {a,b }}$, C. Iemmi ${ }^{\text {c }}$, E.H. Anderson ${ }^{\mathrm{a}, \mathrm{d}}$, W. Chao ${ }^{\mathrm{a}, \mathrm{d}}$, D.T. Attwood ${ }^{\mathrm{a}, \mathrm{d}, \mathrm{e}}$ \\ ${ }^{a}$ NSF Engineering Research Center for Extreme Ultraviolet Science \& Technology, Colorado State University, Fort Collins, CO 80523, United States \\ ${ }^{\mathrm{b}}$ Department of Electrical and Computer Engineering, Colorado State University, Fort Collins, CO 80523, USA \\ c Departamento de Fisica, Facultad de Ciencias Exactas y Naturales, Ciudad Universitaria, Buenos Aires C1428EHA, Argentina \\ ${ }^{\mathrm{d}}$ Center of X-Ray Optics, Lawrence Berkeley National Laboratory, Berkeley, CA 94720, United States \\ ${ }^{\mathrm{e}}$ University of California at Berkeley, Berkeley, CA 94720, United States
}

Available online 25 January 2007

\begin{abstract}
Patterning with extreme ultraviolet light generated by a compact, bright laser source operating at a wavelength of $46.9 \mathrm{~nm}$ is demonstrated using two complementary approaches: multiple beam interferometric lithography and de-magnifying projection. Features with sizes ranging from $370 \mathrm{~nm}$ to $60 \mathrm{~nm}$ were printed in a few seconds in poly-methyl methacrylate resist. These proof-of-principle experiments demonstrate practical table-top nanopatterning tools based on extreme ultraviolet lasers for nanotechnology applications.
\end{abstract}

(c) 2007 Elsevier B.V. All rights reserved.

Keywords: EUV lasers; Table top photolithography; Nanopatterning; Interferometric lithography

The use of short wavelength light has played an important role in the implementation of photolithography tools capable to print arrays of features of a variety of motives with dimensions approaching the wavelength of the illumination. These printing techniques also offer large area coverage and high throughput. In its most widely used geometry, short wavelength light is used to project a mask into a sensitive resist material. The best resolution so far, $28 \mathrm{~nm}$, has been achieved using extreme ultraviolet light from a synchrotron [1]. Array of features with dimensions approaching the wavelength can also be obtained with methods that exploit the coherence of the illumination to generate through the interference of two or more light beams printing of periodic patterns. This approach, named interferometric lithography (IL), can efficiently generate periodic structures with resolution approaching the wavelength of the illumination. Several schemes had been demonstrated using ultraviolet (UV), vacuum-ultraviolet

\footnotetext{
${ }^{*}$ Corresponding author. Address: Department of Electrical and Computer Engineering, Colorado State University, Fort Collins, CO 80523, USA. Tel.: +1970 4918299 .

E-mail address: marconi@engr.colostate.edu (M.C. Marconi).
}

(VUV) laser light illumination, and extreme ultraviolet (EUV) synchrotron radiation to print lines and arrays of holes with different distributions, and as expected by the scaling with wavelengths, with the smallest feature size [2-9].

The recent development of compact and bright table top EUV laser sources provides the opportunity to implement novel patterning tools that would fit on a table-top. An example of these capabilities is the recent demonstration by our group of printing of dense lines in poly methyl metacrylate (PMMA) with a period down to $55 \mathrm{~nm}$ using a IL setup based on a Lloyd's mirror illuminated by a table top $\lambda=46.9 \mathrm{~nm}$ laser [10].

In this paper, we report printing at $\lambda=46.9 \mathrm{~nm}$ of arrays of holes in commercial photoresists using two complementary patterning methods: multiple beam IL and demagnifying projection. With this compact table top nanopatterning tool we were able to print features with sizes of $370 \mathrm{~nm}-60 \mathrm{~nm}$ and areas approximately $50 \times 50 \mu \mathrm{m}^{2}$ in PMMA with exposure times of a few seconds.

The EUV illumination source used in the experiments was a table top capillary discharge laser that emits coherent radiation at $\lambda=46.9 \mathrm{~nm}$ [11]. The laser was configured to 
produce pulses with energy of $\sim 0.4 \mathrm{~mJ}$ and duration of $\sim 1.2 \mathrm{~ns}$ full width at half maximum (FWHM). In this configuration the laser beam has a spatial coherence of $570 \mu \mathrm{m}$ measured at a distance of $1.7 \mathrm{~m}$ from the laser's head. The high degree of monochromaticity of the light with a line width $\Delta \lambda / \lambda \approx 1 \times 10^{-4}$ provides also a very large temporal coherence, of approximately $470 \mu \mathrm{m}$. The high degree of coherence and the large EUV flux makes this source an attractive alternative for IL experiments. The patterning system is completed with a $45 \times 55 \times 40 \mathrm{~cm}^{3}$ vacuum chamber where the experiment is set up. The whole instrument is very compact fitting in an optical table within an area of $0.7 \times 2.6 \mathrm{~m}^{2}$. The EUV laser and the experiment chamber are connected through a vacuum manifold to provide differential pumping of the chamber.

Multiple-beam IL was implemented using a specially fabricated multiple gratings mask composed of a set of four diffraction gratings placed along the sides of a square $50 \times 50 \mu^{2}$, with an arrangement similar as described in [9] and schematically shown in Fig. 1a. The four-gratings mask was mounted at a distance of $150 \mu \mathrm{m}$ from sample such that the four first diffracted orders converge on the same spot, producing an interference pattern with sharp maxima $[12,13]$. The whole assembly sits in the vacuum chamber at a distance of $1.7 \mathrm{~m}$ from the laser head ensuring that the four gratings are overfilled with a laser beam whose output is fully spatially coherent. The diffractive
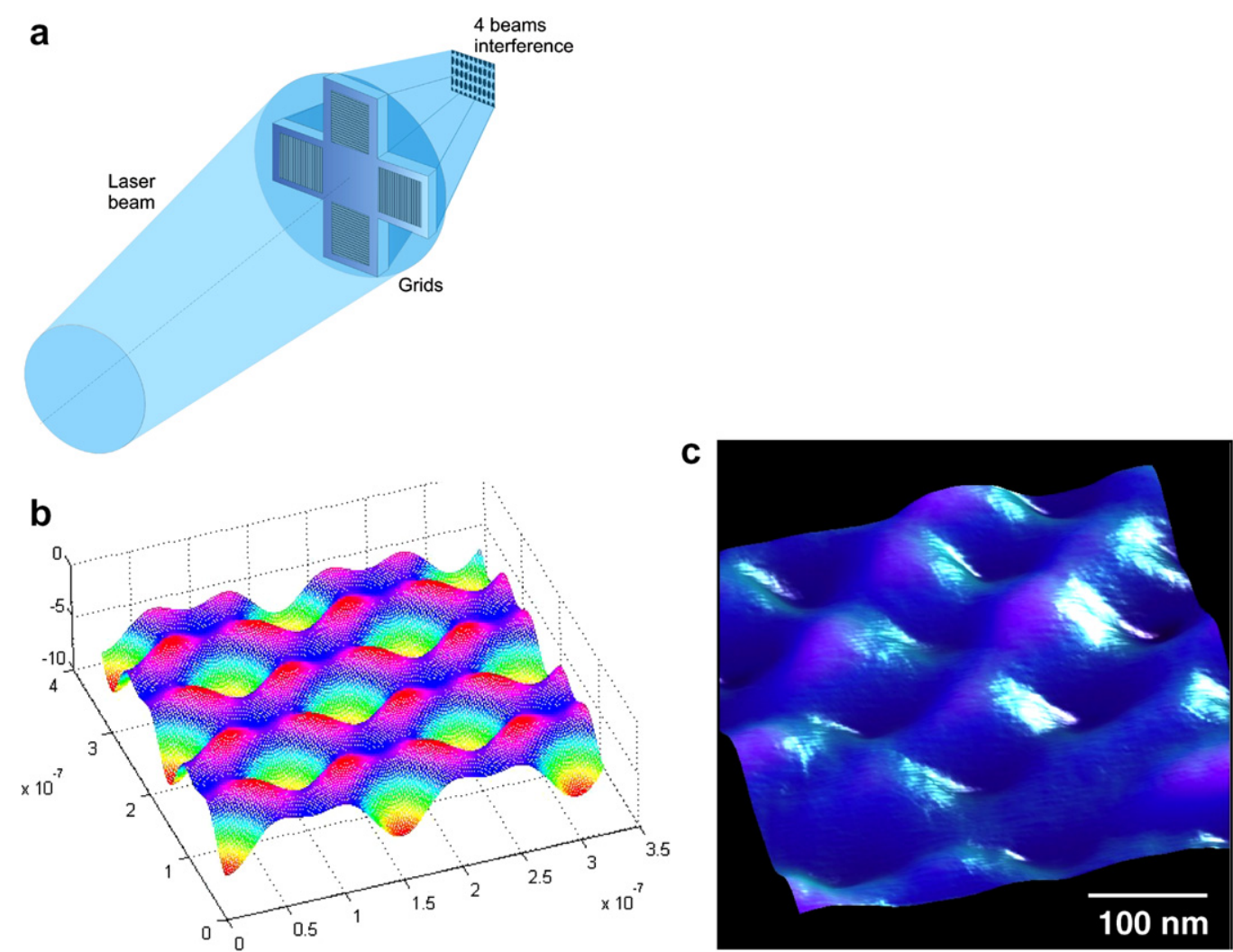

Fig. 1. (a) Experimental set up for interferometric lithography. A diffractive mask composed by 4 crossed diffraction gratings is illuminated by a plane coherent $\lambda=46.9 \mathrm{~nm}$ laser beam producing interference by the superposition of four beams. (b) Calculated hole pattern obtained by illumination with four $\lambda=46.9 \mathrm{~nm}$ non-polarized coherent beams. (c) Atomic force microscope image of the printed pattern obtained in PMMA with $\lambda=46.9 \mathrm{~nm}$ laser illumination. mask was fabricated in a $100 \mathrm{~nm}$ thick silicon nitride membrane using electron beam lithography. The gratings are self standing and consequently required a supporting structure to keep mechanical integrity. Rectangular regions $8 \times 1 \mu \mathrm{m}^{2}$ with $35 \%$ aperture duty cycle, $160 \mathrm{~nm}$ period free standing rulings, were separated by $8 \times 1.6 \mu \mathrm{m}^{2}$ solid supporting bars in the $\mathrm{SiN}_{4}$ membrane.

As shown by Fernandez and Phillion [12], the interference pattern produced by four coherent plane waves impinging on the sample's surface at equal angles depends on the polarization of the beams. For an arbitrary direction of the polarization at the gratings plane, three situations are identified: TE-TE, TE-TM and TM-TM corresponding to the interference resulting from the TE components of the field from each grating pair or from the crossed TE and TM components,

$$
\begin{aligned}
I_{\mathrm{TE}-\mathrm{TE}}(x, y)= & E_{0}^{2}\left[2+\cos \left(2 k y \sin \theta+\varphi_{1}-\varphi_{2}\right)\right. \\
& \left.+\cos \left(2 k x \sin \theta+\varphi_{3}-\varphi_{4}\right)\right] \\
I_{\mathrm{TE}-\mathrm{TM}}\left(x^{\prime}, y^{\prime}\right)= & E_{0}^{2}\left\{2+\cos 2 \theta \cos \left(2 k x^{\prime} \sin \theta\right)\right. \\
& +\cos \left(2 k y^{\prime} \sin \theta+\varphi_{0}\right)+2 \cos \theta \cos \\
& \left.\times\left(k x^{\prime} \sin \theta\right)\left[\cos \left(k y^{\prime} \sin \theta\right)+\cos \left(k y^{\prime} \sin \theta+\varphi_{0}\right)\right]\right\}
\end{aligned}
$$

where $\theta$ in the incidence angle in the sample, $\phi_{i}$ are the relative phases introduced in each of the four diffracted 

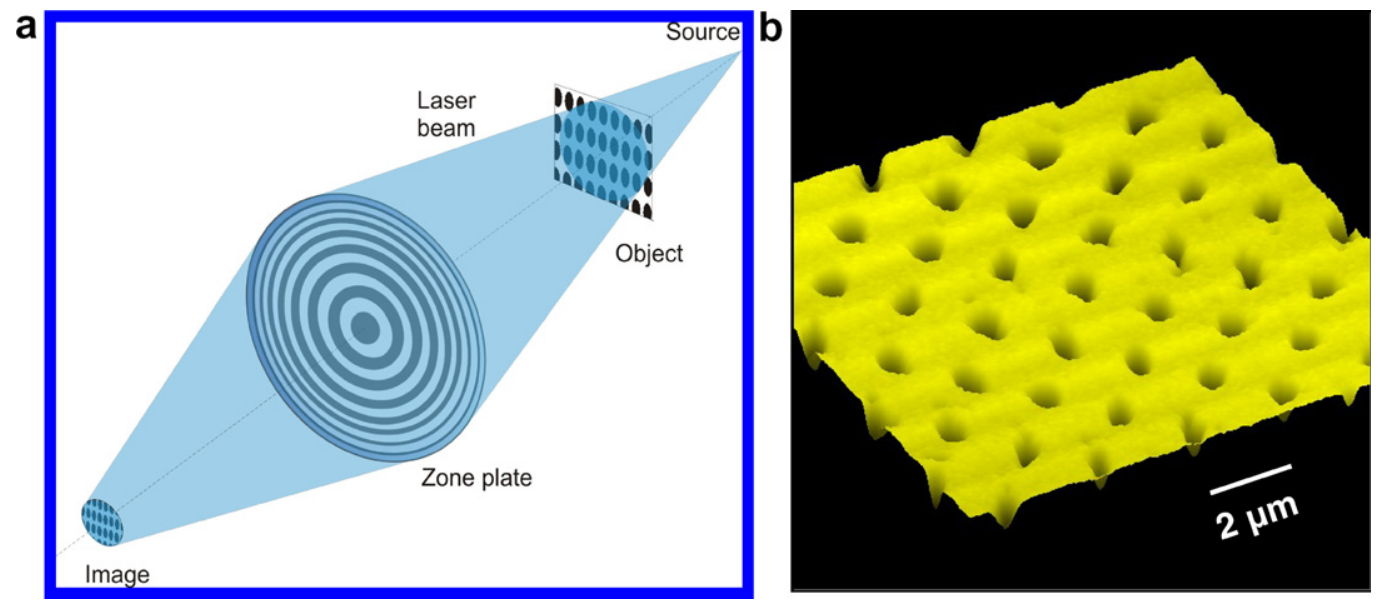

Fig. 2. (a) Scheme of de-magnifying imaging using a self standing zone plate. (b) Atomic force microscope scan of the array of $370 \mathrm{~nm}$ FWHM holes printed over a surface approximately $50 \times 50 \mu \mathrm{m}^{2}$.

beams, $\quad x^{\prime}=x-\left(\phi_{4}-\phi_{3}\right) /(2 k \sin (\theta)), \quad y^{\prime}=y-\left\{\phi_{2}-\right.$ $\left.\left(\phi_{3}+\phi_{4}\right) / 2\right\} /(k \sin (\theta))$ and $\phi_{0}=\phi_{1}+\phi_{2}-\phi_{3}-\phi_{4}$. For the TE-TM polarization a series of holes oriented at $45^{\circ}$ with respect to the two orthogonal polarization axes is obtained. For the TE-TE polarization component, the interference pattern consists of a square array of holes distributed parallel to the polarization axes and with a significantly lower intensity [12]. As the incident angle in the sample approaches to $90^{\circ}$ the TE-TM pattern converges to the TE-TE pattern resulting in a weak interference. However, the TM-TM configuration produces the exact opposite trend with the angle. Since the $\lambda=46.9 \mathrm{~nm}$ laser output is unpolarized, the resulting interference pattern was calculated by assuming an equal contribution from each polarization. The superposition of the diffracted intensity results in a pattern shown in Fig. 1b. In this calculation we also considered that the relative dephasing between the different beams is zero. This can be assured by a proper fabrication of the four gratings diffractive mask. Fig. 1c shows the atomic force microscope scan of the pattern printed in a PMMA coated wafer exposed to the intensity modulation corresponding to the same conditions as simulated in Fig. 1b. The printed pattern is in qualitative good agreement with the calculation and the periodicity and orientation of the holes array is adequately reproduced. The patterned dips in this case have a full width at half maximum (FWHM) of approximately $60 \mathrm{~nm}$ and a total depth of $30 \mathrm{~nm}$, limited by the absorption of the photoresist at $\lambda=46.9 \mathrm{~nm}$. Due to the shadowing provoked by the supporting structure in the self standing gratings, these patterns were obtained over areas of a few microns square.

In a second experiment we utilized a de-magnifying scheme to project a reduced image of a mesh onto the surface of the photoresist. The experimental set up is schematically shown in Fig. 2a. A Ni mesh with $7 \mu \mathrm{m}$ holes separated by $12 \mu \mathrm{m}$ bars was used as the object. This array was projected onto the PMMA coated wafer using a self standing Fresnel zone plate (FZP). The self standing, $0.5 \mathrm{~mm}$ diameter FZP consists of 625 zones with outermost zone width $\Delta r=200 \mathrm{~nm}$. This lens at $46.9 \mathrm{~nm}$ wavelength provides a focal length of $2.14 \mathrm{~mm}$ with a numerical aperture $\mathrm{NA}=0.12$. For these parameters, and assuming the Rayleigh resolution criterion, the spatial resolution of the imaging system is approximately $240 \mathrm{~nm}$ [14]. Fig. $2 \mathrm{~b}$ is an AFM scan of a small section of the pattern area containing holes $\approx 370 \mathrm{~nm}$ full width half maximum. The total area covered with this pattern was over $50 \times 50 \mu \mathrm{m}^{2}$ and was obtained with only $3 \mathrm{~s}$ exposure time.

In summary, we have realized nanometer-scale patterning using a table-top system using two different approaches: IL and de-magnifying imaging. With the former, holes $60 \mathrm{~nm}$ in diameter were printed in PMMA over micron square areas and with exposure times of $50 \mathrm{~s}$. In the latter square holes $370 \mathrm{~nm}$ in width were obtained with exposures of $3 \mathrm{~s}$. These results show that table top EUV lasers have potential to become a useful alternative for printing of periodic nanometer features for nanotechnology applications.

\section{Acknowledgements}

This work was supported under the NER program, NSF Award DMI-0508484 utilizing facilities from the NSF ERC for Extreme Ultraviolet Science and Technology, Award Number EEC-0310717. MGC acknowledges the support through a fellowship from CONICET.

\section{References}

[1] P. Naulleau, K.A. Goldberg, J.P. Cain, E.H. Anderson, K.R. Dean, P. Denham, B. Hoef, K.H. Jackson, IEEE J. Quant. Electron. 42 (2006) 44.

[2] V.N. Golovkina, P.F. Nealey, F. Cerrina, J.W. Taylor, H.H. Solak, C. David, J. Gobrecht, J. Vac. Sci. Technol. B 22 (2004) 99.

[3] H.H. Solak, D. He, W. Li, S. Singh Gasson, F. Cerrina, B.H. Sohn, X.M. Yang, P. Nealey, Appl. Phys. Lett. 75 (1999) 2328. 
[4] H.H. Solak, D. He, W. Li, F. Cerrina, J. Vac. Sci. Technol. B 17 (1999) 3052.

[5] S.H. Zaidi, S.R.J. Brueck, J. Vac. Sci. Technol. B 11 (1993) 658.

[6] H.H. Solak, C. David, J. Vac. Sci. Technol. B 21 (2003) 2883.

[7] T.A. Savas, Satyen N. Shah, M.L. Schattenburg, J.M. Carter, H.I. Smith, J. Vac. Sci. Technol. B 13 (1995) 2732.

[8] M. Switkes, T.M. Bloomstein, M. Rothschild, Appl. Phys. Lett. 77 (2000) 3149.

[9] H.H. Solak, C. David, J. Gobrecht, L. Wang, F. Cerrina, J. Vac. Sci. Technol. B 20 (2002) 2844.
[10] M.G. Capeluto, G. Vaschenko, M. Grisham, M.C. Marconi, S. Luduena, L. Pietrasanta, Y. Lu, B. Parkinson, C.S. Menoni, J.J. Rocca, IEEE Trans. Nanotechnol. 5 (2006) 3.

[11] B. Benware, C. Moreno, D. Burd, J.J. Rocca, Opt. Lett. 22 (1997) 796.

[12] A. Fernandez, D.W. Phillion, Appl. Opt. 37 (1998) 473.

[13] H.H. Solak, C. David, J. Gobrecht, J. Vac. Sci. Technol. B 20 (2002) 2844.

[14] D. Attwood, Soft X-ray and Extreme Ultraviolet Radiation, Principles and Applications, Cambridge University Press, 2000. 